\title{
Strong Interaction Studies with PANDA at FAIR
}

\author{
Karin Schönning ${ }^{1, \star}$ for the PANDA collaboration \\ ${ }^{1}$ Dept. of Physics and Astronomy, Uppsala Universitet, Box 516, S-75120 Uppsala, Sweden
}

\begin{abstract}
The Facility for Antiproton and Ion Research (FAIR) in Darmstadt, Germany, provides unique possibilities for a new generation of nuclear-, hadron- and atomic physics experiments. The future PANDA experiment at FAIR will offer a broad physics programme with emphasis on different aspects of hadron physics. Understanding the strong interaction in the perturbative regime remains one of the greatest challenges in contemporary physics and hadrons provide several important keys. In these proceedings, PANDA will be presented along with some high-lights of the planned physics programme.
\end{abstract}

\section{Introduction}

The Standard Model (SM) of particle physics has been proven successful in describing elementary particles and their interactions. Strong interactions of quarks and gluons are described by the theory of Quantum ChromoDynamics (QCD) [1]. At high energies, the coupling $\alpha_{s}$ is sufficiently weak to enable a perturbative treatment (pQCD) that has been rigorously and successfully tested [2]. However, at low and intermediate energies, the strong coupling $\alpha_{s}$ grows and pQCD breaks down. Strongly interacting particles at this scale therefore emerge as hadrons where the quarks and gluons are confined within a radius of $\approx 1 \mathrm{fm}$. Understanding the strong interaction at energies in the confinement domain up to $\mathrm{pQCD}$ is one of the most challenging questions in contemporary physics. More specifically, this can be considered a puzzle containing many pieces, where some of the most important are

- Why and how are quarks confined into hadrons?

- How is mass generated by the strong interaction? Only $1 \%$ of the proton mass originates from the current quark masses.

- What is the origin of the so-called spin crisis? Only $1 / 3$ of the proton spin originates from the current quarks.

- Are there exotic hadrons, i.e. non- $q \bar{q}$ or non-qqq states? Which forces act between their constituents?

- What is the role of strangeness and charm in systems with larger degrees of complexity, e.g. nuclei or even neutron stars?

Hadrons are excellent probes of the strong interaction. The scale of the interaction within a hadron is given by the mass of its quarks. For strange hadrons, the scale is governed by the mass of the strange quark, i.e. $m_{s} \approx 100 \mathrm{MeV}$. This is close to the QCD cut-off, $\Lambda_{Q C D}$, which is the scale at which

^e-mail: karin.schonning@physics.uu.se 
quarks confine into hadrons. Strange hadrons therefore provide a key to confinement. The charm scale is more than ten times larger since the mass of the charm quark is $m_{c} \approx 1300 \mathrm{MeV}$. Here, the strong coupling is $\alpha_{s} \approx 0.3$ which is just barely outside the region where pQCD applies. Charmed hadrons therefore provide a bridge between perturbative and non-perturbative QCD. To summarize, strange and charmed hadron physics probe the strong interaction at two different energy scales and a systematic comparison could be very illuminating.

Furthermore, the study of hadrons in the precision frontier shed light on other open questions within the Standard Model, for example: i) Are there baryon decays that violate CP conservation? ii) What are the hadronic contributions to $\mathrm{SM}$ precision observables, e.g. the anomalous magnetic moment of the muon, $g-2$ ?

The future PANDA experiment at FAIR in Darmstadt, Germany, will be a unique facility to study a wide range of aspects of the strong interaction. PANDA will be optimized for strange and charmed hadron physics. In the following, we will present the design and outline its broad physics programme.

\section{The PANDA experiment}

The PANDA (anti-Proton ANnihilation in DArmstadt) detector will be an integral part of the HESR (High Energy Storage Ring). The antiproton beam will impinge on a fixed target which will provide a high luminosity environment crucial for the physics programme. The HESR will provide anti-protons with momenta from of $1.5 \mathrm{GeV} / \mathrm{c}$ up to $15 \mathrm{GeV} / \mathrm{c}$, corresponding to centre-of-mass energies of 2.0 up to $5.5 \mathrm{GeV}$. The HESR will have two operation modes: the high resolution mode and the high luminosity mode. The high resolution mode will provide a beam momentum precision up to $5 \cdot 10^{-5}$ thanks to stochastic and electronic cooling. The luminosity will be $\approx 10^{31} \mathrm{~cm}^{-2} \mathrm{~s}^{-1}$. The high luminosity mode offers a 20 times higher luminosity, $\approx 2 \cdot 10^{32} \mathrm{~cm}^{-2} \mathrm{~s}^{-1}$ and a beam momentum resolution of $10^{-4}$ from stochastic cooling. During the first few years of PANDA operation, the so-called Modularized Start Version (MSV) will be available. It will provide a luminosity of $\approx 10^{32} \mathrm{~cm}^{-2} \mathrm{~s}^{-1}$ and a beam momentum resolution of $10^{-4}$. The target will be either of cluster-jet type or frozen hydrogen pellet type for the $\bar{p} p$ annihilation programme. For $\bar{p} A$ studies, target foils will be used. PANDA will cover a solid angle of almost $4 \pi$ and consist of a Target Spectrometer (TS) and a Forward Spectrometer (FS). The TS will detect particles emitted at polar angles larger than $10^{\circ}$ in the horisontal plane and larger than $5^{\circ}$ in the vertical plane. The MicroVertex Detector (MVD) will surround the target region and provide precise vertex position measurements. The vertex space resolution will be of the order $50 \mu m$ in $\mathrm{x}$ and $\mathrm{y}$ and $100 \mu \mathrm{m}$ in z. Gas Electro Multiplier (GEM) planes and a Straw Tube Tracker (STT) will provide precise tracking $\left(\Delta p_{T} / p_{T}=1.2 \%\right.$ when combined with the MVD) of charged particles and a 2T solenoid magnet. A barrel Detection of Internally Reflected Cherenkov Light (DIRC) and a Time-of-Flight (TOF) detector will discriminate between pions, kaons and protons and an Electro Magnetic Calorimeter (EMC) equipped with $17200 \mathrm{PbWO}_{4}$ crystals will provide state-of-the-art photon reconstruction and separation between electrons and pions. Muon detectors surrounding the solenoid magnet will provide identification of muons. The FS will cover angles below $10^{\circ}$ in the horizontal plane and below $5^{\circ}$ in the vertical plane. It will comprise a Forward Tracking System (FTS) with three pairs of straw tube planes. A dipole magnet bend the charged particle trajectories. Furthermore, an Aerogel Ring Imaging Cherenkov Counter (RICH) and a forward time-of-flight (FTOF) will enable particle identification and a Forward ElectroMagnetic Calorimeter (FEMC) photon reconstruction and electron/pion separation. Also in the FS there will be a muon detector. The PANDA experiment will utilize a new, time-based data acquisition that reads out the data continously from the stream. This put high demands on the readout techniques and the foreseen software trigger that will be used for this purpose, but it also opens up new possibilities regarding readout rates and data storage. The PANDA detector, shown in Figure 2, is described in detail in Ref. [3]. 


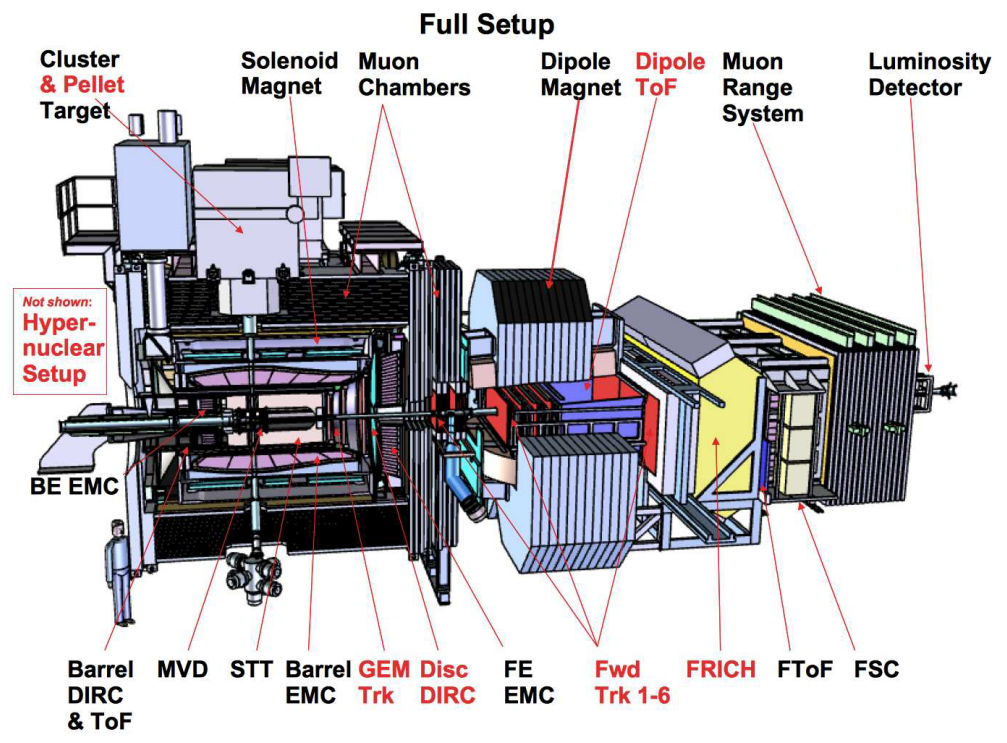

Figure 1. View of the future PANDA detector.

\section{Nucleon structure}

The proton was discovered almost 100 years ago and together with the neutron, it constitutes the major part of the visible mass of the universe. However, a complete understanding of its structure is yet to be found. As mentioned before, only $1 \%$ of its mass and $1 / 3$ of its spin can be explained by the simple quark model. The quark structure is revealed by reactions where absorbtion, radiation or exchange of real or virtual photons. Such processes dominate in reactions involving charged leptons in the inital and/or final state. A multitude of experimental efforts devoted to study different aspects of nucleon structure are ongoing world-wide. The majority are these (e.g. at COMPASS, HERMES, JLAB) utilize electromagnetic probes and thereby they explore the space-like (SL) region of the nucleon structure observables. PANDA, on the other hand, will utilize $\bar{p} p$ annihilations which means that time-like (TL, positive momentum transfer $q^{2}$ ) observables will be explored: i) Generalized Distribution Amplitudes (GDAs), which are accessible by wide angle Compton scattering in hard exclusive processes like e.g. $\bar{p} p \rightarrow \gamma^{*} \gamma$ and $\bar{p} p \rightarrow \gamma^{*} \pi^{0}$, ii) Transistion Distribution Amplitudes (TDAs) from e.g. $\bar{p} p \rightarrow \gamma^{*} M \rightarrow e^{+} e^{-} M$ or $\bar{p} p \rightarrow \gamma^{*} M \rightarrow J / \Psi M$ where $M=\pi^{0}, \eta, \omega, \rho^{0} \ldots$ [4], iii) Transverse Momentum distributions (TMDs) in Drell-Yan processes, i.e $\bar{p} p \rightarrow \gamma^{*} X \rightarrow \mu^{+} \mu^{-} X$ and iv) Electro Magnetic Form Factors (EMFFs) in $\bar{p} p \rightarrow \gamma^{*} \rightarrow l^{+} l^{-}$and $\bar{p} p \rightarrow \gamma^{*} \pi^{0} \rightarrow l^{+} l^{-} \pi^{0}$, where $l=e, \mu$.

In the following we will discuss the proton EMFFs. For protons, the differential cross section of the $\bar{p} p \rightarrow \rightarrow l^{+} l^{-}, l=e, \mu$ process can be parameterized in terms of the electric $G_{E}$ and the magnetic $G_{M}$ form factors assuming one-photon exchange (see for example [5]). $G_{E}$ and $G_{M}$ are complex function of the momentum transfer squared $q^{2}$ and the moduli $\left|G_{E}\right|$ and $\left|G_{M}\right|$ can be extracted from the total and differential cross sections. Questions that are addressed in nucleon EMFF studies are in general: i) How does the ratio $\mathrm{R}=G_{E} / G_{M}$ depend on $q^{2}$ ? Existing data from PS170 [6] differ from BaBar [7] and BES III [8] at low $q^{2}$. ii) Do the EMFFs agree with the predictions from pQCD in the high $q^{2}$ limit? iii) Down to which $q^{2}$ is the pQCD picture valid? iv) Do TL and SL EMFFs agree in the high $q^{2}$ limit, as predicted by pQCD and analyticity [9]? Existing high $q^{2}$ data from BaBar [7] indicates a difference in the TL and SL magnetic FFs by a factor of 2. 
Recently, simulation studies of the $\bar{p} p \rightarrow e^{+} e^{-}$reaction with PANDA have been carried out [10]. When the high luminosity mode of the HESR is available, the EMFFs can be determined with an unprecedented precision within $\left.5.4<q^{2}<13.9(\mathrm{GeV} / c)^{2}\right)$. Furthermore, the so-called effective form factor (see e.g. [7]) can be measured up to $q^{2}<29(\mathrm{GeV} / c)^{2}$. The very large expected background from $e . g . \bar{p} p \rightarrow \pi^{+} \pi^{-}$could be suppressed by a factor $\sim 10^{8}$. Another on-going simulation study show good prospects also for form factor measurement using the $\bar{p} p \rightarrow \mu^{+} \mu^{-}$reaction [11].

Since the form factors $G_{E}$ and $G_{M}$ are complex in the TL region, they have a relative phase. The phase introduces polarization effects [12]. It is presently being investigated whether an upgraded version of PANDA could comprise a polarized target. This would enable the very first measurement of the phase of the proton EMFFs.

\section{Hyperon dynamics}

The key question in hyperon physics is "What happens if you replace one (or several) light quark(s) in the nucleon with one (or several) heavier one(s)?" The strange quark is sufficiently light to relate the knowledge about hyperons to nucleons and vice versa. In other words, hyperon physics provide a new angle to increase our understanding of the structure of the nucleon. At the same time the strange quark is heavy enough to be traced in the reactions. In that way one can obtain new information about the structure of baryons which is not accessible in the pure light ( $u$ and $d$ ) sector. The clearest situation is achieved in exclusive hyperon-antihyperon production.

The scale probed in such reactions is governed by the strange-quark mass, i.e. $m_{s} \approx 100 \mathrm{MeV}$. Since this corresponds to the confinement scale, the relevant degrees of freedom are unclear - quarks and gluons, or hadrons? The production of strange hyperons therefore probes QCD in the intermediate region between the perturbative and non-perturbative regime. Besides effective field theories, which can account for the universal aspects of the long-range forces, phenomenological models are needed, which require guidance by experimental data.

In particular, spin observables have been proven decisive in testing different models for polarizations and spin correlations [13-15]. Spin observables, e.g. the polarization, are straight-forward to measure for ground-state hyperons thanks to their weak, self-analyzing decays. This means that the decay products are preferentially emitted along the direction of spin of the parent hadron. In the case of $\Lambda \rightarrow p \pi^{-}$, the angular distribution of the proton is related to the $\Lambda$ polarization by $I\left(\cos \theta_{p}\right)=\frac{1}{4 \pi}\left(1+\alpha_{\Lambda} P_{n} \cos \theta_{p}\right)$, where $\alpha_{\Lambda}=0.64$ [2] is the asymmetry parameter. In production of spin 1/2 hyperons $Y$ in $\bar{p} p \rightarrow \bar{Y} Y$ with unpolarized beam and target, the polarization of the hyperon and the anti-hyperon and the spin correlation between them are accessible.

Some hyperons decay into other hyperons, e.g. the $\Xi$. In the $\Xi^{-} \rightarrow \Lambda \pi^{-}, \Lambda \rightarrow p \pi^{-}$process, the additional asymmetry parameters $\beta$ and $\gamma$ of the $\Xi^{-}$hyperon are accessible via the joint angular distribution of the $\Lambda$ hyperons and the protons [16].

For spin $\frac{3}{2}$ hyperons, e.g. the $\Omega^{-}$, the spin structure is more complicated. Here, we consider the polarization parameters of individual spin $\frac{3}{2}$ hyperons only. Spin $3 / 2$ hyperons produced in strong processes like $\bar{p} p \rightarrow \overline{\Omega^{+}} \Omega^{-}$have seven non-zero polarization parameters. Three of these can be extracted from the angular distribution of the decay baryon, e.g. $\Omega^{-} \rightarrow \Lambda K^{-}$. The remaining four can be obtained by studying the joint angular distribution $I\left(\theta_{\Lambda}, \phi_{\Lambda}, \theta_{p}, \phi_{p}\right)$ of the $\Lambda$ hyperons from the $\Omega^{-}$ decay and the protons from the subsequent $\Lambda$ decay.

The PS185 collaboration have provided a large set of high-quality data on single-strange hyperons [17], which triggered various theoretical investigations [13-15]. The ambition is to survey the spin structure of $\bar{p} p \rightarrow \bar{Y} Y$ for all ground-state multi-strange and single-charmed hyperons. 
Table 1. Results from simulation studies of the various production reactions of ground state hyperons as well as the excited $\Xi^{-*}(1820)$. The efficiencies are exclusive, i.e. all final state particles are reconstructed.

\begin{tabular}{llllll}
\hline$p_{\bar{p}}(\mathrm{GeV} / c)$ & Reaction & $\sigma(\mu \mathrm{b})$ & Eff $(\%)$ & Decay & Rate at $10^{31} \mathrm{~cm}^{-2} \mathrm{~s}^{-1}$ \\
\hline 1.64 & $\bar{p} p \rightarrow \bar{\Lambda} \Lambda$ & 64 & 10 & $\Lambda \rightarrow p \pi^{-}$ & $28 s^{-1}$ \\
\hline 4 & $\bar{p} p \rightarrow \bar{\Lambda} \Sigma^{0}$ & $\approx 40$ & 30 & $\Sigma^{0} \rightarrow \Lambda \gamma$ & $30 s^{-1}$ \\
\hline 4 & $\bar{p} p \rightarrow \bar{\Xi}^{+} \Xi^{-}$ & $\approx 2$ & 20 & $\Xi^{-} \rightarrow \Lambda \pi^{-}$ & $2 s^{-1}$ \\
\hline 12 & $\bar{p} p \rightarrow \bar{\Omega}^{+} \Omega^{-}$ & $\approx 0.002^{*}$ & $\approx 30$ & $\Omega \rightarrow \Lambda K^{-}$ & $\approx 4 h^{-1}$ \\
\hline 12 & $\bar{p} p \rightarrow \bar{\Lambda}_{c}^{-} \Lambda_{c}^{+}$ & $\approx 0.1^{*}$ & $\approx 30$ & $\Lambda_{c} \rightarrow \Lambda \pi^{+}$ & $\approx 2 d^{-1}$ \\
\hline 4.6 & $\bar{p} p \rightarrow \bar{\Xi}^{+} \Xi^{-*}(1820)$ & 1 & 5 & $\Xi^{-*} \rightarrow \Lambda K^{-}, \Lambda \rightarrow p \pi^{-}$ & $15000 d^{-1}$ \\
\hline
\end{tabular}

Simulation studies of exclusive hyperon production have been performed using a simplified Monte Carlo framework [18-20]. The estimated signal rates are given in Table 1. They are calculated assuming the Day One luminosity of $10^{31} \mathrm{~cm}^{-2} \mathrm{~s}^{-1}$. The only well known cross section is $\bar{p} p \rightarrow \bar{\Lambda} \Lambda$ at $1.64 \mathrm{GeV} / c$; the other single- and double-strange cross sections are obtained by extrapolating data collected at other energies. For $\Omega$ and $\Lambda_{c}$ production in $\bar{p} p$ annihilations, no data exist. The quoted values for $\sigma\left(\bar{p} p \rightarrow \bar{\Omega}^{+} \Omega^{-}\right)$and $\sigma\left(\bar{p} p \rightarrow \bar{\Lambda}_{c}^{-} \Lambda_{c}^{+}\right)$are theoretical predictions [21]. From the simulations we conclude that already with the Day One setup, the spin observables of multi-strange baryons can be thoroughly explored for the first time. The full setup will extend these studies to charmed hyperons. As the FAIR facility matures, studies of $\mathrm{CP}$ violation in baryon decays will become possible, for instance in double-strange decays such as $\Xi^{-} \rightarrow \Lambda \pi^{-}[19]$.

\section{Baryon spectroscopy}

Hadron spectroscopy provides another laboratory in which to explore the dynamics of strongly interacting quarks and gluons confined into hadrons. In baryon spectroscopy, the focus lies on multistrange baryons. In light and strange baryon spectroscopy we search for answers the following questions: i) to which extent do the excitation spectra of baryons consisting of $u, d, s$ follow the systematics of SU(3) flavour symmetry? ii) which degrees of freedom are relevant for the excitation modes of baryons? iii) how important is the dynamics in baryon-meson systems? iv) are there exotic baryon states, e.g. pentaquarks or dibaryons? Baryon spectroscopy has been decisive in the development of our understanding of the microscopic world, the best example being the formulation of the quark model. In the dawn of particle physics during 1950's and 1960's, the concept of quarks was not yet known. Thanks to extensive experiments, a plethora of new particles were observed and it soon became clear that they could not all be elementary. Many were found to have a finite life-time and were classified as strange. The new-found particles could be organized according to "the eight-fold way" or SU(3) symmetry and as a result, the $\Omega^{-}$hyperon was predicted. The discovery of the $\Omega^{-}$ baryon [22] demonstrated the success of the eight-fold way and shortly after, the quark model was formulated by Gell-Mann and Zweig [23]. The quark model, where three equivalent valence quarks interacting flavour-independently and color-magnetically with one-gluon exchange, has been proven successful in classifying ground-state baryons and describing some of their static properties. The intense worldwide effort in baryon spectroscopy has been focused on $N^{*}$ and $\Delta$ resonances. Most of the known baryon resonances have masses smaller than $2 \mathrm{GeV} / c^{2}$ and were discovered in elastic $\pi N$ scattering experiments. In recent years, many laboratories worldwide (JLab, ELSA, MAMI, GRAAL, Spring-8 etc) have studied these resonances in electromagnetically induced reactions ([24, 25]). The observed spectra show many features that cannot be explained within the quark model. For example, 
the predicted so-called level ordering of baryonic states with positive and negative parity disagree with the experimental findings. Another problem found by the experiments is that of missing resonances: predicted baryonic states that haven't been observed by experiment. One possible explanation is that the states haven't been found because they couple weakly to $\pi N$ and $\gamma N$. They would as a consequence be unlikely to be produced in pion- or photoproduction. Searches using different probes, e.g. anti-protons, could shed light on this puzzle.

Another explanation of the missing states is that they are simply not there, which in turn would indicate that our understanding of baryon structure is incomplete. It would be very illuminating to see whether the features of the light baryon spectra are carried over to the strange sector, i.e. the excited single-, double- and triple-strange hyperon spectra. That would enable a systematic comparison of systems containing different strangeness. The existing data bank is however scarce, in particular regarding double- and triple-strange hyperons. Only one excited $\Xi$ state and zero excited $\Omega$ states are considered well established with the PDG classification system. Ongoing efforts at JPARC and JLAB could open up for studies of light double strange hyperons, i.e. $\Xi^{*}$. Excited baryons can also be studied in charmonium och bottom baryon decays, but the detectors are not optimized for exclusive multi-strange hyperon studies. Thus, the prospects with currently running facilities for heavier doubleand triple-strange remain scarce. This is in contrast to the spectroscopy of bottom $(b)$ and charmed (c) baryons is a very active field of research today thanks to numerous B-factories like BaBar, BELLE and LHCb. To summarize, current experimental facilities worldwide study either light baryons or charmed and bottom hyperons while leaving a gap in the strange sector.

In order to investigate the prospect of PANDA being able to fill this gap, a simulation study of the $\bar{p} p \rightarrow \bar{\Xi}^{+} \Xi^{-*}(1820)+c . c$. has been carried out [26]. The $\Xi^{-*}(1820) \rightarrow \Lambda K^{-}$was considered and a BR of $100 \%$ was assumed. For a cross section of $\approx 1 \mu \mathrm{b}$ and the Day One luminosity $10^{31} \mathrm{~cm}^{-2} \mathrm{~s}^{-1}$, an exclusive detection rate of $\approx 15000$ events per day is expected, as shown in Table 1 . This demonstrates that PANDA indeed has the potential of filling the gap in the strange sector from Day One.

\section{Charmonium spectroscopy}

A long-standing question in hadron physics concerns the existence of so-called exotics, i.e. states which are inconsistent with the meson $(q \bar{q})$ or baryon $(q q q)$ picture. QCD allows for e.g. multiquarks $(q q \overline{q q}$ or $q q q q \bar{q})$, glueballs ( $g g$ or $g g g$ ) or hybrids $(q \bar{q} g)$ but until recently, the evidence have been scarce. In the past, many efforts were made in the light sector (for reviews see for example Refs. $[27,28])$ where unambiguous identification is often difficult due to the states being broad, largely overlapping and mixing. The charm sector has proven more straight-forward in the sense that the observed states are relatively narrow and well separated. Recently, the discovery of the first charged charmonium state $Z_{c}^{ \pm}(3900)$ by BES III [29] and and confirmed by Belle [30] implied a major breakthrough and led to an increased activity in the field. Another interesting example is the $J^{P C}=1^{++}$ state $X(3872)$, that have puzzled the scientific community for more than a decade $[31,32]$. The nature of the $X(3872)$ is disputed: is it a multiquark or a $D \bar{D}^{*}$ molecule, or maybe an more conventioal $\chi_{c 1}(2 P)$ candidate, with some $D \bar{D}^{*}$ threshold features? In order to solve this puzzle, a precision measurement of its width and line-shape is required. However, most running or planned facilities have clear disadvantages that limit their scopes. Most of them are either i) $e^{+} e^{-}$colliders (BES III and Belle II), where direct formation of any state that is not $J^{P C}=1^{--}$is suppressed, or ii) high energy $p p$ experiments $(\mathrm{LHCb})$, where any charmonium-like state needs to be formed in production together with several tagged or untagged recoils. In both cases, one relies on the detector resolution when determining the properties of the $X(3872)$. With the PANDA experiment, the antiproton beam enables direct formation of any $q \bar{q}$-like state $X$ in $\bar{p} p \rightarrow X$. The width and the line-shape of any such 
state can therefore be measured in an energy scan where the precision is only limited by the precision of the beam momentum, which will be around $10^{-4}$ with the high luminosity mode and $5 \cdot 10^{-5}$ with the high precision mode. A recent simulation study [33] show that the width can be measured with a sensitivity of $\Gamma / \Delta \Gamma>5$ for widths between 90 and $120 \mathrm{keV}$. Furthermore, a simulation of the molecular line-shape show that it will be possible to distinguish between a virtual and a bound state. These measurements can be carried out also using the MSV with 80 days of data taking.

\section{Other physics topics}

The scope of PANDA is very broad thanks to the antiproton beam and the versatile detector. Important physics topics that will be studied, but were not discussed here, are for instance open charm physics, hypernuclear physics and hadrons in nuclear matter.

\section{Summary}

PANDA will be a unique experiment with an excellent potential to shed light on many of the most important puzzles of contemporary physics. In the following, high-lights of the scope are outlined as a function of time.

\section{Day One}

Already during the start-up year of PANDA, with a reduced HESR luminosity $\left(10^{31} \mathrm{~cm}^{-2} \mathrm{~s}^{-1}\right)$ and a staged version of the detector, PANDA can provide unprecedented precision of the $X(3872)$ width and a first measurement of the $X(3872)$ line-shape. Furthermore, PANDA can collect uprecedented data samples on spin observables of single-strange hyperons and the first measurement ever of doublestrange spin observables in $\bar{p} p$ annihilations. PANDA can access the full spectrum of single- and double-strange excited hyperons with a unique statistical precision.

\section{First Years}

During the following years, the experimental situation will be the same as the first year but since more data will be ackumulated over time, the physics scope widens. PANDA will for the first time measure the seven accessible polarization parameters of the $\Omega^{-}$hyperon and provide full triple-strange excited hyperon spectrum. PANDA will further explore the precision frontier of charmonium spectroscopy. In addition, the modular, versatile design of PANDA opens up for unique contributions to hypernuclear physics.

\section{Long-term plans}

The design luminosity of the HESR $\left(2 \cdot 10^{32} \mathrm{~cm}^{-2} \mathrm{~s}^{-1}\right)$ and the complete PANDA detector setup will enable high precision studies of nucleon structure, e.g. proton EMFFs. Furthermore, open charm can be studied with world-leading statistical precision. The expected unprecedented hyperon samples will enable searches for CP violation in baryon decays.

\section{References}

[1] Fritzsch H et al., Phys. Lett. B47 365 (1971); Politzer H D, Phys. Rev. Lett. 301346 (1973); Gross D J and Wilczek F, Phys. Rev. Lett. 301343 (1973); Weinberg S, Phys. Rev. Lett. 31494 (1973).

[2] Olive K A et al. (Particle Data Group) Chin. Phys. C38 090001(2014).

[3] The PANDA collaboration, Technical Progress Report (2005).

[4] The PANDA collaboration, Eur. Phys. J. A 51107 (2015).

[5] Zichichi A et al., Nuovo Cim. 24170 (1962). 
[6] Bardin G et al. Nucl. Phys. B 4113 (1994).

[7] Lees J P et al. Phys. Rev. D 88 7:072009 (2013).

[8] Ablikim M et al. Phys. Rev. D 91 11:112004 (2015).

[9] S. Pacetti et al. Phys. Rep. 550-551 1 (2015).

[10] The PANDA collaboration, "Feasibility studies of time-like proton electromagnetic form factors at PANDA at FAIR” submitted 2016 (arXiv:1606.01118 hep-ex).

[11] Zimmermann I, Talk at the ECT* workshop Probing transverse nucleon structure at high momentum transfer (2016)

[12] Dubnickova A et al. Nuovo Cim. 109241 (1996).

[13] Kohno M and Weise W, Phys. Lett. B 17915 (1986); Rubinstein H R and Snellman H Phys. Lett. B 165187 (1985); Furui S and Faessler A, Nucl. Phys. A 486669 (1987); Burkardt M and Dillig M Phys. Rev. C 371362 (1988); Alberg M A et al. Z. Phys. A 331207 (1988).

[14] Tabakin F and Eisenstein R A Phys. Rev. C 311857 (1985); Kohno M and Weise W, Phys. Lett. B. 17915 (1985); La France P et al. Phys. Lett. B 214317 (1988); Timmermans R G E et al. 1992 Phys. Rev. D 452288 (1992); Haidenbauer J et al. Phys. Rev. C 462516 (1992).

[15] Ortega P G et al. Phys. Lett. B. 696352 (2011).

[16] Koch W Analysis of scattering and decay ed. Nikolic M (New York-London-Paris:Gordon and Breach, 1968).

[17] Johansson T 2003, proceedings of 8th Int. Conf. on Low Energy Antiproton Physics 95 (2003).

[18] The PANDA collaboration, Physics Performance Report (2009).

[19] Thomé E, "Multi-Strange and Charmed Antihyperon-Hyperon Physics for PANDA" (Ph. D. Thesis, Uppsala University, 2012).

[20] Grape S, "Studies of PWO Crystals and Simulations of the $\bar{p} p \rightarrow \bar{\Lambda} \Lambda, \bar{\Lambda} \Sigma^{0}$ Reactions for the PANDA experiment” (Ph.D. Thesis, Uppsala University, 2009).

[21] Kaidalov A B and Volkovitsky P E Z, Phys. C 6351 (1994); Titov A I and Kämpfer B, Phys. Rev. C 78025201 (2008); Gornitschnig A T et al. Eur. Phys. J. A 4243 (2009); He J et al. Phys. Rev. D 84114010 (2011); Khodjamirian A et al. Eur. Phys. J. A 4831 (2012).

[22] Barnes V E et al., Phys. Rev. Lett. 12204 (1964).

[23] Gell-Mann M, Phys. Lett. 8214 (1964); Zweig G, CERN-preprint CERN-TH-401 402 (1964).

[24] Klempt E and Richard J M, Rep. Prog. Phys. 821095 (2010).

[25] Crede V and Roberts W, Rep. Prog. Phys. 76076301 (2013).

[26] Pütz J, Proceedings of FAIRNESS 2016, Garmisch-Partenkirchen, Germany (2016).

[27] Crede V and Meyer C A, Prog. Part. Nucl. Phys. 6374 (2009).

[28] Meyer C A and Swanson E, Prog. Part. Nucl. Phys. 8221 (2015).

[29] Ablikim M et al., Phys. Rev. Lett. 110252001 (2013).

[30] Liu Z Q et al. Phys. Rev. Lett. 110252002 (2013).

[31] Abe K et al. Phys. Rev. Lett. 91262001 (2003).

[32] Aaij et al. Phys. Rev. Lett. 110222001 (2013).

[33] Nerling F, talk at the 11th International Workshop on Heavy Quarkonium (2016) 